\title{
Towards Civility: Efforts to Address Harassing Behaviors in Engineering
}

\author{
Teri K. Reed, Teri J. Murphy, Cijy Elizabeth Sunny, Whitney Gaskins, and Ashley Paz y Puente
}

\section{In 2018, the National Academies} of Sciences, Engineering, and Medicine (NASEM) published the report, Sexual Harassment of Women: Climate, Culture, and Consequences in Academic

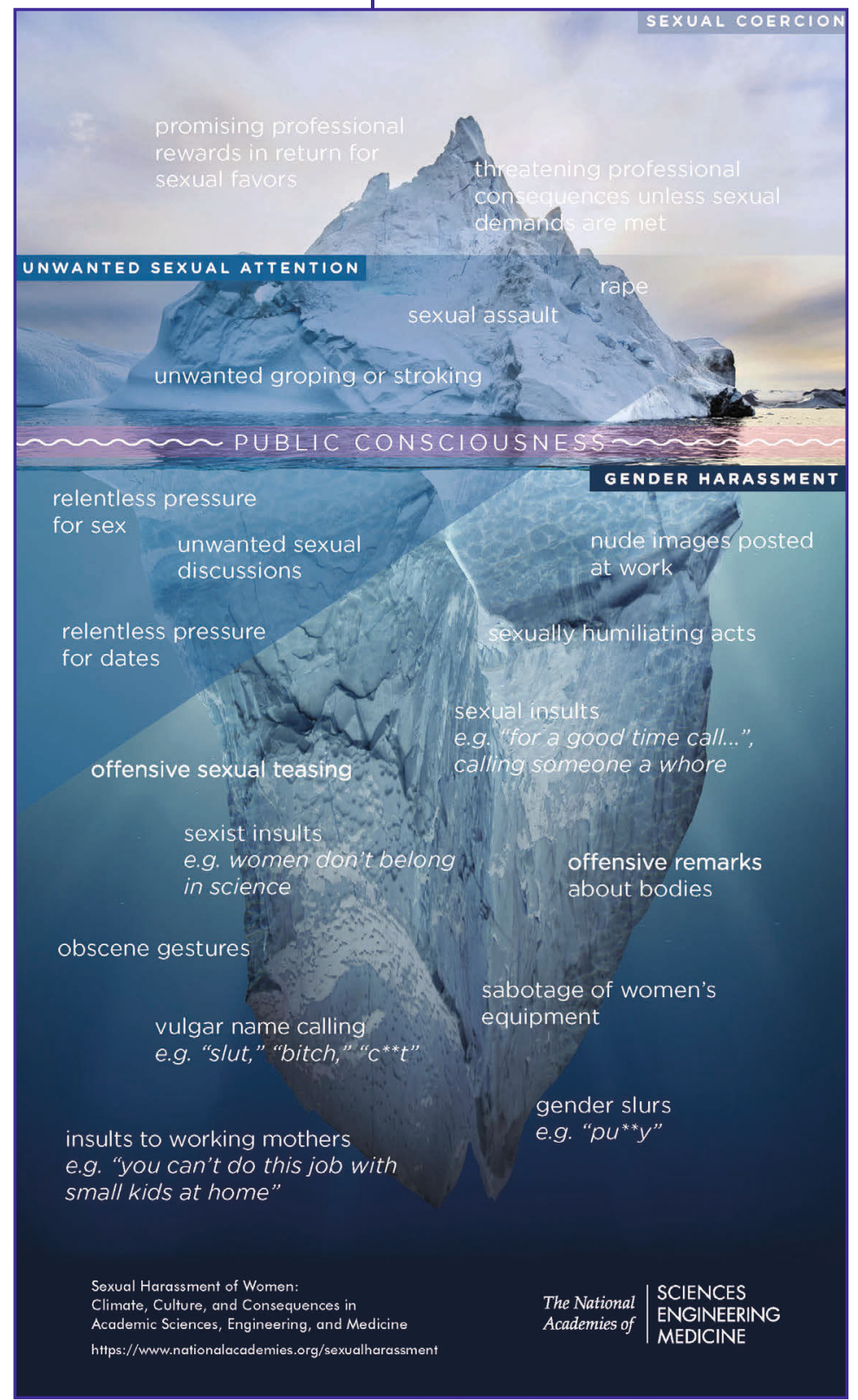

Figure 1. The public consciousness of sexual harassment and specific sexually harassing behaviors. ${ }^{1}$
Sciences, Engineering, and Medicine. ${ }^{1}$ As with other NASEM consensus studies, the report provides a state-of-the-art synthesis and evaluation of the knowledge base and offers recommendations both for practice and for further research. The report categorizes sexually harassing behaviors into:

1. Gender harassment (verbal and nonverbal behaviors that convey hostility, objectification, exclusion, or second-class status about members of one gender);

2. Unwanted sexual attention (verbal or physical unwelcome sexual advances, which can include assault); and

3. Sexual coercion (when favorable professional or educational treatment is conditioned on sexual activity). ${ }^{1}$

As illustrated in Figure 1 (often referred to as the Iceberg Model), sexual coercion and unwanted sexual attention are more visible in the public awareness, but gender harassment is actually the most prevalent of the three categories. Evidence presented in the report reveals that academia is second only to the military in prevalence of sexually harassing behaviors ( $58 \%$ vs. $69 \%$ ) and that within academia, engineering is second only to medicine ( $25 \%$ vs. $45 \%) .{ }^{1}$ Although the NASEM report focuses on academia, a related body of research indicates that "women's accounts of internships and summer jobs differ from that of men. They describe a culture that is isolating, that often assumes women are second-class experts, and where sexism is normative."

A team in the College of Engineering \& Applied Science (CEAS) at the University of Cincinnati (UC) has been working from several directions to replace harassing behaviors with civility: fundamental research, promising practices for training, and continued commitment to action through institutional membership in a NASEM action collaborative.

\section{Fundamental Research}

Focus on Experiential Learning: As the founder of co-op more than 100 years ago, UC has a long and rich history of engaging students in co-op and other experiential learning 


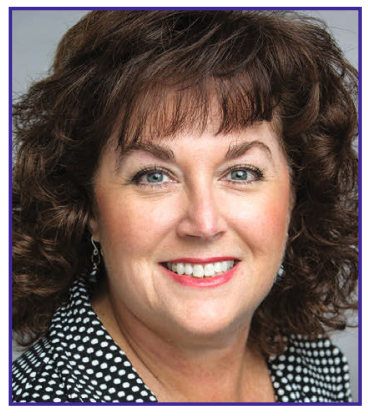

Teri K. Reed

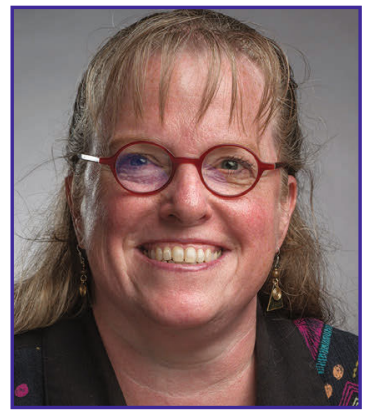

Teri J. Murphy

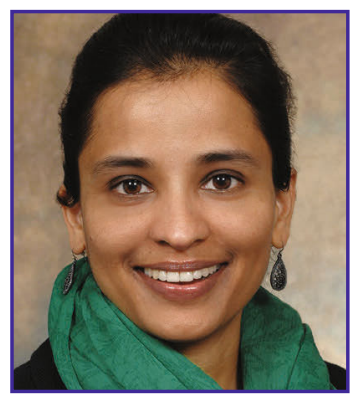

\section{Cijy Elizabeth Sunny}

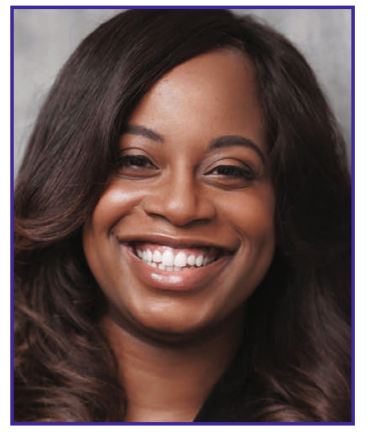

\section{Whitney Gaskins}

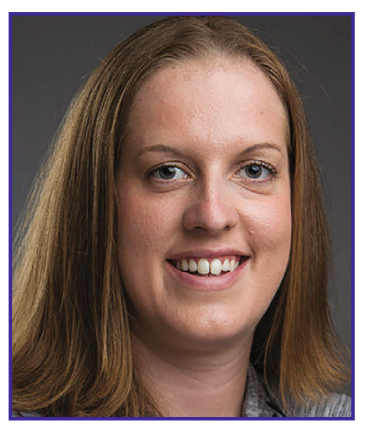

Ashley Paz y Puente opportunities, which positions us to pay special attention to student experiences in these settings and the impact of those experiences. Thus, in our research, we distinguish between classroom settings (i.e., an environment in a formal learning setting, including labs and field trips, with multiple students and an instructor[s]) and experiential learning settings (e.g., co-op, study abroad, undergraduate research, service learning).

Preliminary Results: In the initial (current) phase of our mixed-methods research design, we adapted the Administrator Researcher Campus Climate Collaborative (ARC3) survey into an instrument that we have called the UC Civility Audit. ${ }^{3-4}$ Of particular note, we added a module focused on encounters in experiential learning settings. The UC Civility Audit 2020, completed by 112 undergraduate engineering students, provides initial baseline prevalence estimates and informs subsequent phases of the research. This response sample is overrepresented in female students and students from racial/ethnic populations underrepresented in engineering (e.g., Black, Latinx, Native American). Preliminary survey results indicate that of the 23 who experienced sexist or sexually offensive language, gestures, or pictures (which are forms of gender harassment) in experiential learning settings, 19 were female and four were male. Examples of comments shared by participants in openended items include:

- "made sexual comments about women in general and loose remarks about me and other women at the company" (fifth-year, mechanical engineer)

- 'I just don't like people asking me if I'm gay or not" (first-year, computer science)

- "a snide remark was made regarding if I knew what I was doing because I was working with tools" (third-year, biomedical engineer)

- "especially in construction, engineering, and more dominated fields, women have to deal with more degrading comments" (fourthyear, civil engineer)

Responses indicated that the majority of these behaviors (13 of the 23) came from a colleague or coworker. As described by one participant: "During my first co-op, a coworker repeatedly made harassing comments and jokes about me and towards me daily for a month until I told him to stop. The comments became less frequent after that, but they were still made" (second-year, chemical engineer).

What's Next: Gaps in the Data: The

NASEM report and other resources (including our own experience with the UC Civility Audit 2020) identify a number of challenges in determining prevalence estimates: $^{1}$

- Response rates to climate surveys are low.

- Response rates from individuals who identify as underrepresented and/or LGBTQ+ are even lower.

- The low response rates also mean that intersectionality is difficult to study.

- Data from climate surveys are rarely disaggregated sufficiently to examine discipline-specific issues.

The next phases of our research design will address some of these challenges. Specifically, we will conduct a group level assessment (GLA), which will help us translate the needs and priorities of stakeholders (e.g., industry, students, professors, university administration) into the design and implementation of interventions. Then, combined results from the UC Civility Audit 2020 and the GLA will inform phenomenological studies that use interviews and focus groups to develop deeper understandings of both shared and individual experiences.

\section{Promising Practices for Training}

Faculty/Staff: Each new cohort of faculty, joining CEAS in any one fiscal year, participate in orientations that cover many topics, including culture in the classroom/lab. While this training includes topics such as being a mandatory reporter for Title IX, it also covers bystander interventions. Using role play, each faculty is paired with a peer, alternating playing the roles of student and faculty, to go through scenarios from known incidents. Role play is an active learning technique that simulates reality 
in an environment in which negative effects are minimized and confidence to respond increases.

Students: In August 2018, the UC Department of Engineering Education conducted a two-day orientation for $\sim 60$ students (graduate and undergraduate) preparing to serve as teaching assistants for the Introduction to Engineering Design Thinking course (taken by $\sim 1,400$ firstyear engineering majors). In addition to the two hours of Title IX and Clery Act training required for compliance, the orientation included a three-hour session on diversity, equity, and inclusion and a 90-minute session on bystander intervention. The bystander intervention training, which was conducted by the assistant director for the UC Student Wellness Center, focused on bystander intervention in the classroom (versus, for example, at a party). Modifications of this session were included for subsequent cohorts of teaching assistants either during the orientation or as part of the weekly teaching team meetings throughout the semester.

What's Next: Upstander Intervention

Training: What separates a bystander from an upstander is the response behaviors of a witness to a harassing behavior situation involving perpetrator(s) and target(s). An upstander is moved to action both in an individual (courageous, action-oriented, assertive, compassionate, and leader) and collective (part of a community, relationship to others in this community, responsibility, partnership opportunities, and shared concerns) perspective. As emphasized in a training offered by the National Institutes of Health, evolving from bystander to upstander means engaging to diffuse a situation, interrupt the situation, and keep a record of the event as evidence. ${ }^{5}$

\section{Continued Commitment to Action through Institutional Membership in a NASEM Action Collaborative}

In 2019, UC became an institutional member of the NASEM Action Collaborative on Preventing Sexual Harassment in Higher Education. The university's internal working group structure will parallel the structure developed by NASEM: data/evaluation (measuring climate and gauging progress on campus); prevention (initiatives to prevent harassment); response (responding to harassment when it occurs); and remediation (limiting the damage caused by sexual harassment). The NASEM report includes 15 recommendations for institutions of higher education and funding agencies. ${ }^{1}$ In line with a number of these recommendations, our work is intended, long-term, to determine and track the prevalence and nature of harassing behaviors in engineering, especially gender harassment; implement promising practices for the prevention of harassing behaviors; and institutionalize a culture of civility and respect.

\section{Endnotes}

1. National Academies of Sciences, Engineering, \& Medicine (NASEM), "Sexual Harassment of Women: Climate, Culture, and Consequences in Academic Sciences, Engineering, and Medicine" (Washington, D.C.: The National Academies Press, 2018). https://doi.org/10.17226/24994

2. C. Seron, S.S. Silbey, E. Cech, and B. Rubineau, "Persistence Is Cultural: Professional Socialization and the Reproduction of Sex Segregation," Work and Occupations 43(2), 178-214 (2016).

3. C.E. Sunny, T.K. Reed, T.J. Murphy, and W. Gaskins, "Towards Civility: Understanding and Confronting Harassing Behaviors Through Mixed Research Approaches in Engineering Learning Settings," Proceedings of the 2020 Frontiers in Education Conference, Uppsala, Sweden, October 2020.

4. K.M. Swartout, W.F. Flack, S.L. Cook, L.N. Olson, P.H. Smith, and J.W. White, "Measuring Campus Sexual Misconduct and Its Context: The AdministratorResearcher Campus Climate Consortium (ARC3) Survey," Psychological Trauma: Theory, Research, Practice, and Policy, 11(5), 495-504 (2019). https://doi. org/10.1037/tra0000395

5. "Moving from Bystander to Upstander: Take Action to Combat Harassment and Aggression" [video file], (National Institutes of Health (NIH), 2020), https:// videocast.nih.gov/summary. asp?live $=37765$. Accessed 26 May 2020.
Teri K. Reed is a professor of chemical engineering with a courtesy appointment in engineering education and is the assistant vice president of faculty research development for the Office of Research at the University of Cincinnati (UC).

Teri J. Murphy is a professor in the UC Department of Engineering Education.

Cijy Elizabeth Sunny is a postdoctoral researcher in the UC Department of Engineering Education.

Whitney Gaskins serves as assistant dean of inclusive excellence and community engagement at the UC College of Engineering and Applied Science.

Ashley Paz y Puente is a professor of mechanical and materials engineering at $U C$ and, as a TMS member, is actively involved in the Diversity, Equity, and Inclusion Committee. 